\title{
An Objective Assessment to Investigate the Impact of Turning Angle on Freezing of Gait in Parkinson's Disease
}

\author{
Matilde Bertoli*§, Andrea Cereatti*\$†, Ugo Della Croce*§ and Martina Mancini ${ }^{\ddagger}$ \\ Email: \{mbertoli,acereatti,dellacro\}@uniss.it_mancinim@ohsu.edu \\ *PolComIng Department, University of Sassari, Sassari, Italy \\ $\S$ Interuniversity Centre of Bioengineering of the Human Neuromusculoskeletal System, Sassari, Italy \\ 'Department of Electronics and Telecommunications, Politecnico di Torino, Turin, Italy \\ Department of Neurology, Oregon Health and Science University, Portland, OR
}

\begin{abstract}
Freezing of gait (FoG) is often described in subjects with Parkinson's disease (PD) as a sudden inability to continue the forward walking progression. FoG occurs most often during turning, especially at sharp angles. Here, we investigated 180 and 360 degrees turns in two groups: PD subjects reporting FoG (FoG+), and PD subjects without FoG (FoG-). Forty-three subjects (25 FoG+, 18 FoG-) wore an inertial sensor on their back while walking back and forth continuously for 2 min (reversing direction with a $180^{\circ}$ turn), and while turning in place for $1 \mathrm{~min}$ (alternating $360^{\circ}$ turning in opposite directions). Objective measures (turn duration, peak velocity, jerkiness and range of acceleration) were computed during the turns and compared across FoG+ and FoG- groups. Results showed that FoG+ compared to FoG- took significantly a longer time to complete $360^{\circ}$ turns than $180^{\circ}$ turns. A significant lower turn peak velocity, higher jerkiness and an increased range of medio-lateral acceleration was also found in FoG+. Significant differences between the two groups across the two turning tasks validated the hypothesis that sharper turns might cause higher instability in FoG+ compared to FoG-.
\end{abstract}

Keywords-PD, FoG, turn, inertial sensor

\section{INTRODUCTION}

Freezing of gait $(\mathrm{FoG})$ is one of the most disabling features in Parkinson's Disease (PD). It is associated with increased risk of falls, it interferes with daily activities and it substantially affects quality of life [1]. FoG denotes the "absence or marked reduction of the forward progression of the feet, despite the intention to walk" [2]. FoG is an episodic phenomenon of still controversial pathophysiology. However, FoG episodes are usually triggered by postural transitions, such as step initiation or turning, or by environmental constraints, such as passing through a narrow path or being under time pressure [3], [4]. Specifically, it has been shown that turning may be the most effective task in provoking FoG [5]. In fact, turning is a challenging motor task, requiring a coupling between anticipatory postural adjustments and scaling of walking [2].

Generally, subjects with PD walk slowly with short stride length, even more so prior to and during a change in walking

Grants: NIH Career Development Award K99 HD078492 01A1 \& R00 HD078492 (Mancini). direction. Subjects with PD experiencing FoG (freezers) during $180^{\circ}$ turns exhibit a different motor strategy compared to healthy controls and non freezers (reduced step width, reduced medial deviation and increased forward progression of the center of mass) [6]. In addition, it has been observed that subjects with PD display greater variability at sharper turns (i.e turning at larger angle), and sharper turns tend to particularly elicit freezing episodes [7], [8]. Snijders et al., and Mancini et al., showed that repeated $360^{\circ}$ turns on the spot were more effective in provoking FoG compared to $180^{\circ}$ turns during walking [5], [9]. $360^{\circ}$ turn is indeed a task demanding a fine postural control tuning, and it has been hypothesized that FoG may be related to a lack of initiation of postural adjustments [10]. Moreover, in a stepping in place task FoG was associated with rapid, small and inefficient weight transfers, possibly due to mismatched gains in the perception and execution of the movement [11].

Recently, wearable sensors have increasingly been used for instrumented clinical evaluations to obtain quantitative outcomes. Small inexpensive inertial sensors are becoming a powerful tool to perform motion analysis due to their ease of use. Instrumented motor tests are nowadays widely accepted [12]. In particular, the assessment of turning with inertial sensors allows investigating complex locomotor patterns and gathering additional insights on motor control [13].

Several studies ([6], [7], [9], [14], [15]) demonstrated that objective measures from a turning task can differentiate between PD patients and healthy controls, and highlighted the need for further research to focus on the clinical relevance of such measures. In particular, as also suggested in Visser et al., it would be interesting to correlate yaw angular velocity during turning to clinical measures as those for FoG, as this approach might be useful to evaluate the outcome of intervention studies aimed at improving FoG [14]. Furthermore, investigating the underlying mechanism of FoG is important to advice an effective rehabilitation intervention.

To this purpose, we developed and applied a method, based on the use of a single inertial sensor on the lower trunk, to perform a quantitative gait analysis in presence of $180^{\circ}$ and $360^{\circ}$ turns in freezers and non freezers PD populations ( $\mathrm{FoG}+$ and FoG-). 


\section{MATERIAL AND METHODS}

\section{A. Participants}

Forty-three subjects with PD were recruited through the Parkinson's Center of Oregon clinic at Oregon Health \& Science University (OHSU). All gave informed, written consent to a protocol approved by OHSU's Institutional Review Board. Individuals were excluded if they could not walk seven meters without walking aids, or if they had any neurological disease (other than PD), musculoskeletal or vestibular disorder, and dementia.

Subjects were divided in two groups based on their answer to the first question of the New FoG Questionnaire (NFOG-Q): "Have you experienced FoG in the past month?". Twenty-five $(19 \mathrm{M}, 6 \mathrm{~F})$ answered 'yes' and were classified as freezers (FoG+), and eighteen (14M, 4F) were assigned to the FoGgroup. FoG + mean age was $69 \pm 7$ years, and disease severity was rated $45.7 \pm 12$ as per the motor section of the Movement Disorder Society-sponsored revision of the Unified Parkinson's Disease Rating Scale (MDS-UPDRS-III). FoG- mean age was

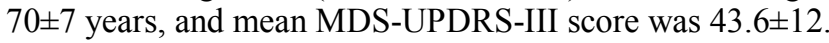

\section{B. Experimental Setup}

Testing was carried out in the practically defined OFF state (12+ hours of PD medication withdrawal). At the beginning of the testing session, a trained examiner administered the motor section of the MDS-UPDRS to quantify disease severity and the NFOG-Q to assess the perceived severity of FoG.

An inertial sensor (Opal - APDM, Inc) was positioned on the back approximately at the level of L5. Subjects then performed two motor tasks: $180^{\circ}$ and $360^{\circ}$ turns. The $180^{\circ}$ turns were part of a two minutes long walk, where the subject walks straight for 7 meters at a comfortable speed, turns back and keep walking in the opposite direction. No indications were given about direction of turning or strategy. For the $360^{\circ}$ turns, the subject turned in place, starting from a standing position, $360^{\circ}$ clockwise, and then $360^{\circ}$ counter-clockwise, repeating this sequence for one minute.

The reference frame of the inertial sensor was oriented approximately along the three human body anatomical directions. An estimate of its orientation with respect to the global frame was provided by an on-board Kalman filter. The signals from the Opal sensor were recorded at $128 \mathrm{~Hz}$, streamed wirelessly to a laptop and stored for subsequent offline analysis with Matlab (MathWorks. R2016a).

\section{Data Analysis}

Inertial sensor data were automatically segmented to detect turns. To this purpose, two different algorithms were implemented to identify the $180^{\circ}$ and $360^{\circ}$ turns.

For the $180^{\circ}$ turns, the algorithm was based on previous work from [16]. The angular velocity was expressed in the global coordinate system and its vertical component low pass filtered (Butterworth, $1.5 \mathrm{~Hz}$ cutoff frequency). The offset was then removed (by subtracting the mean of the signal during the first 3 seconds, during which the subject was standing still). Candidate turns were detected as vertical angular velocity peaks higher than $15 \%$, and for each peak the preceding and following $5 \%$ s threshold crossing were set as instants of turn beginning and ending. Additional checks were performed on the candidate turns in order to isolate the $180^{\circ}$ turn. First of all, turns in the same direction separated by less than $0.1 \mathrm{~s}$ were merged. Then, turns lasting less than $0.5 \mathrm{~s}$ or more than $10 \mathrm{~s}$ were discarded. Finally, the relative turn angle was computed integrating the vertical angular velocity over the turn duration and, when resulting less than $45^{\circ}$, lead to the turn elimination.

For the $360^{\circ}$ turns, a novel algorithm was implemented based on an approach exploiting local magnetic field inversion and angular velocity [17]. The two planar components (AP and ML) of the magnetometer signals were low pass filtered (Butterworth, $1 \mathrm{~Hz}$ cutoff frequency), and their sum was computed. The mean value computed during the first $3 \mathrm{~s}$ from this composed signal was removed, and a moving average (windows length $0.5 \mathrm{~s}$ ) was used for smoothing. Prototype turns were detected as peaks higher than $70 \%$ of the signal max value and further apart than $3 \mathrm{~s}$, and for each peak the preceding and following $20 \%$ threshold crossing were used to isolate the turn. For each prototype turn, the zero-crossings of the filtered, offset-free vertical angular velocity were used as turn beginning and ending instants. An additional control was performed in case of an incorrect merging of two consecutive turns: the turning angle was computed integrating the vertical angular velocity absolute value over the turn duration and, when resulting greater than $400^{\circ}$, lead to the turn division.

For each detected turn (for both trials, see example in Fig. 1), the following objective measures were computed: turn duration, peak angular velocity, jerkiness and range of acceleration. Specifically, turn duration (s) was measured as the interval of time from the beginning to the ending of the turn.

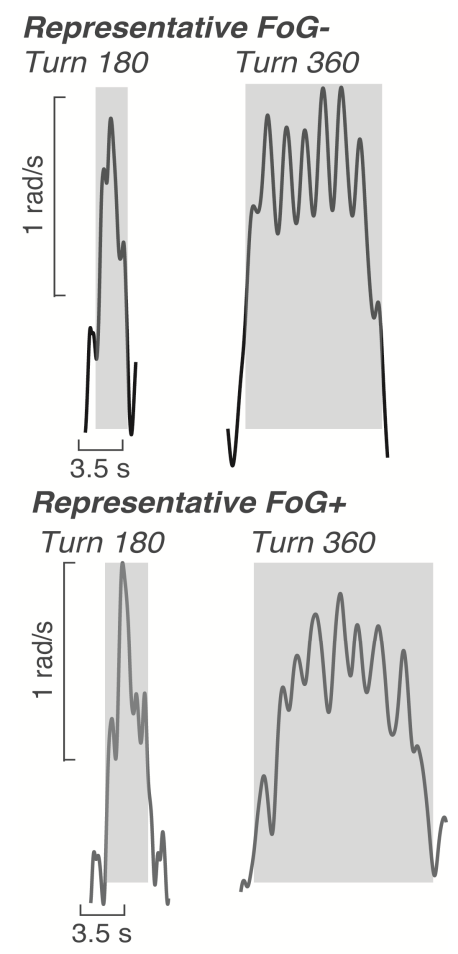

Fig. 1. Time series of trunk angular velocity profiles during the 180 and 360 turning tasks in a FoG- (upper panel) and a FoG+ (lower panel). In FoG+ the time needed to complete the turns is longer than in FoG-. 
Turn peak velocity $(\%)$ was defined as the vertical angular velocity maximum peak amplitude. Turn jerk $\left(\mathrm{g}^{2} / \mathrm{s}\right)$, time derivative of acceleration, was used to quantify fluidity of turning in both antero-posterior (AP) and medio-lateral (ML) directions. Turn range $\left(\mathrm{m} / \mathrm{s}^{2}\right)$ was also computed for both ML and AP accelerations. At first the metrics where averaged grouping them by turning direction (most affected side vs. least affected side), but since there was no evident difference and their distribution was overlapping, the average between sides for each subject was used for the statistical analysis. A twoway (groups $\times$ task) repeated measures analysis of variance (ANOVA) was used to investigate the difference in the metrics between groups $(\mathrm{FoG}+/ \mathrm{FoG}-)$ and within tasks $\left(180^{\circ} / 360^{\circ}\right)$. Since turn jerk and turn duration distributions were not normal, for the ANOVA analysis they were transformed in logarithmic scale. SPSS (IBM V.23) was used to run statistical analyses.

\section{RESULTS}

Table I summarizes the mean (STD) of each turn measure across groups and the ANOVA results, significant differences $(\mathrm{p}<0.05)$ are indicated in bold.

A significant group, task, and group $\mathrm{x}$ task interaction effect was observed for turn duration and both AP- and ML- jerks. Turn duration was longer in the $360^{\circ}$ task compared to the $180^{\circ}$ task in both groups (significant task effect, $\mathrm{F}=173.09$, $\mathrm{p}<0.001$ ), Fig. 2. In addition, FoG + took a longer time to complete the turn compared to FoG- both during the $180^{\circ}$ (2.68s FoG- ; 3.40s FoG + ) and the $360^{\circ}$ task $(5.59 \mathrm{~s}$ FoG-; $9.42 \mathrm{~s} \mathrm{FoG}+)$, with a significant group by task effect $(\mathrm{F}=6.99$, $\mathrm{p}=0.01$ ). AP- and ML- jerks were significantly higher in the $360^{\circ}$ task compared to the 180 task $(\mathrm{F}=30.42, \mathrm{p}<0.001$; and $\mathrm{F}=70.59, \mathrm{p}<0.001$, respectively) with $\mathrm{FoG}^{+}$showing a significantly higher increase in turn jerk during the $360^{\circ}$ task compared to $180^{\circ}$ (significant interaction effect, AP: $\mathrm{F}=6.18$, $\mathrm{p}=0.02 ; \mathrm{ML}: \mathrm{F}=9.36, \mathrm{p}<0.001)$.

A significant group effect was found for turn peak velocity, which was lower in FoG $+(\mathrm{F}=15.86, \mathrm{p}<0.001)$, and there was a significant trend $(\mathrm{F}=5.09, \mathrm{p}=0.03)$ towards lower peak velocity during $360^{\circ}$ task within $\mathrm{FoG}+$ (mean peak velocity remains almost constant in FoG- in $180^{\circ}$ and $360^{\circ}$ task, while it decreases in $\mathrm{FoG}+$ ). No significant task effect was observed for turn peak velocity.

Neither AP- nor ML- ranges were significantly different across groups. For AP-Range there was a significant task effect (greater AP-Range for the $180^{\circ}$ task), but no group by condition effects. Instead, ML-range showed a significant task and interaction effect (greater ML-range during $360^{\circ}$ compared to $180^{\circ}$ within $\mathrm{FoG}+$ ).

\section{DISCUSSION}

The aim of this study was to quantitatively characterize $180^{\circ}$ and $360^{\circ}$ turns in subjects with PD to investigate the impact of turning angle on FoG.

The FoG+ group took longer time to complete turns, performing them at slower speed, with greater jerkiness, but similar range of acceleration. Every metric, except turn peak velocity, showed a significant task effect, indicating a difference in the $360^{\circ}$ turn compared to the $180^{\circ}$ turn. The significant interaction effects, in all the turning metrics but APrange, supports the hypothesis that the $\mathrm{FoG}+$ group showed different turning behaviors depending on the turning task.

Specifically, the increase in turning duration from $180^{\circ}$ to $360^{\circ}$ was larger in the FoG + group. This result is consistent with [8], in which the FoG+ group was found to walk more slowly than healthy controls and FoG- during a $180^{\circ}$ turn, and even more so in the $360^{\circ}$ turn. A potential explanation for such increase is that FoG + showed freezing episodes in the $360^{\circ}$ task but not in the $180^{\circ}$ task, leading to an increase in turning duration.

A similar pattern was observed for ML- and AP- jerks. In fact, the increase in AP- and ML- jerks from $180^{\circ}$ to $360^{\circ}$ turn was larger in the $\mathrm{FoG}+$ group. This difference in turning smoothness could be the result of a larger number of steps needed to complete a turn in the FoG+ group, or of more FoG episodes. Further analysis will investigate the association between the amount of freezing and the metrics presented here.

The turn peak velocity was similar among turning tasks, but interestingly, the $\mathrm{FoG}+$ group tended to slow down during the $360^{\circ}$ turn compared to the $180^{\circ}$ while FoG- showed a similar turn peak velocity in both turn tasks. This is therefore suggesting that the $\mathrm{FoG}+$ subjects have more difficulties in completing the $360^{\circ}$ turn compared to the $180^{\circ}$ turn. This finding is in accordance with our previous results characterizing the $360^{\circ}$ turn, where the $\mathrm{FoG}+$ group showed a lower peak speed than the FoG- (and healthy controls).

TABLE I. SUMMARY OF THE OBJECTIVE MEASURES IN SUBJECTS WITH (FOG+) AND WITHOUT (FoG-) FREEZING OF GAIT.

\begin{tabular}{|c|c|c|c|c|c|c|c|}
\hline Measure & Group & $180^{\circ}$ mean (STD) & $360^{\circ}$ mean $(\mathrm{STD})$ & & Group & Task & Interaction \\
\hline \multirow{2}{*}{ duration (s) } & $F_{O G-}$ & $2.68(0.54)$ & $5.59(4.09)$ & F-value & 16.93 & 173.09 & 6.99 \\
\hline & $F_{O G+}$ & $3.40(0.72)$ & $9.42(4.36)$ & p-value & 0.00 & 0.00 & 0.01 \\
\hline \multirow{2}{*}{ peak velocity $\left({ }^{\circ} \mathrm{s}\right)$} & $F o G-$ & $131.48(31.11)$ & $133.22(39.21)$ & F-value & 15.86 & 2.81 & 5.09 \\
\hline & $F_{O G+}$ & $104.51(19.82)$ & $92.73(26.82)$ & $\mathrm{p}$-value & 0.00 & 0.10 & 0.03 \\
\hline \multirow{2}{*}{ ML-jerk ( $\left.\mathrm{g}^{2} / \mathrm{s}\right)$} & $F o G-$ & $0.080(0.02)$ & $0.106(0.03)$ & F-value & 8.02 & 70.59 & 9.36 \\
\hline & $\mathrm{FoG}^{+}$ & $0.092(0.03)$ & $0.174(0.09)$ & p-value & 0.01 & 0.00 & 0.00 \\
\hline \multirow{2}{*}{ AP-jerk $\left(\mathrm{g}^{2} / \mathrm{s}\right)$} & $F o G-$ & $0.065(0.02)$ & $0.077(0.03)$ & F-value & 7.00 & 30.42 & 6.18 \\
\hline & $\mathrm{FoG}^{+}$ & $0.076(0.03)$ & $0.116(0.05)$ & $\mathrm{p}$-value & 0.01 & 0.00 & 0.02 \\
\hline \multirow{2}{*}{ ML-range (g) } & $F o G-$ & $0.40(0.08)$ & $0.40(0.11)$ & F-value & 0.25 & 4.48 & 4.81 \\
\hline & $F_{O G+}$ & $0.38(0.08)$ & $0.44(0.013)$ & p-value & 0.62 & 0.04 & 0.03 \\
\hline \multirow{2}{*}{ AP-range (g) } & $F o G-$ & $0.32(0.07)$ & $0.28(0.08)$ & F-value & 0.59 & 14.24 & 0.61 \\
\hline & $\mathrm{FoG}^{+}$ & $0.30(0.07)$ & $0.27(0.05)$ & p-value & 0.45 & 0.00 & 0.44 \\
\hline
\end{tabular}




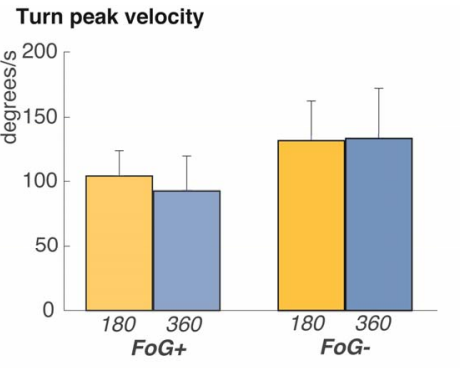

Turn duration
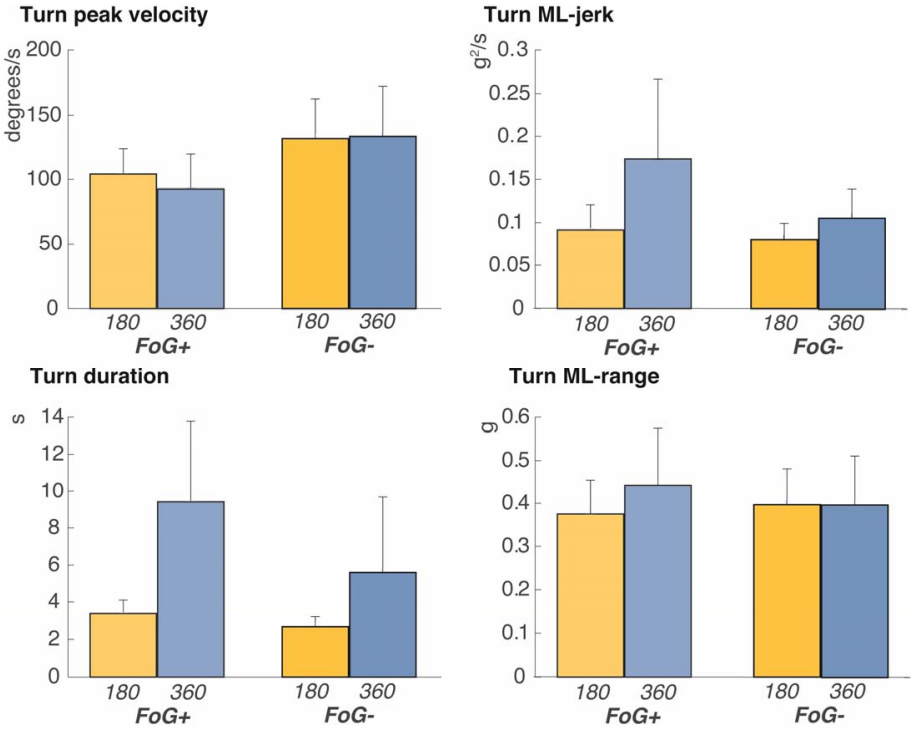

Fig. 2. Mean and SEM of the objective measures. The greatest chages can be observed in the FoG + during the $360^{\circ}$ turn (decrease in velocity, and increase in ML-jerk, ML-range and duration).

The ML-range of acceleration during turn showed a task and a group $\mathrm{x}$ task interaction effect, suggesting that only FoG+ have a greater $\mathrm{ML}$ acceleration in the $360^{\circ}$ turn compared to the $180^{\circ}$ turn. Interestingly, few studies ([6], [11]) showed either a greater ML center of mass displacement in FoG+ compared to FoG-, or a reduced ML weight shifting during a repetitive stepping in place task. Our results did not show significant differences between the two groups for ML-range of acceleration, however we did not analyze separately turns with and without FoG episodes.

AP-range did not show an interaction effect, but it showed a strong task effect $(p<0.001)$. The intrinsic motor task characteristics, walking and turning versus turning in place, could explain such difference. In fact, in the $180^{\circ}$ task the subjects are progressing forward before the turn, instead for the $360^{\circ}$ task subjects are more prone to shift their weight laterally to complete the task. This difference in the experimental set-up in measuring $180^{\circ}$ and $360^{\circ}$ turn is also a limitation of the present study.

Nonetheless, our previous assertions do not lose their validity. Our findings support the hypothesis that turning at a sharper angle may pose more difficulties for $\mathrm{FoG}^{+}$, possibly due to an increasing demand on bilateral coordination [8].

These results can be useful to develop algorithms for generating a biofeedback aimed at alleviating or preventing FoG prior challenging motor tasks. The findings here reported can be also relevant for the outcome evaluation after interventions aimed at reducing FoG episodes, or to devise optimal and more effective rehabilitation treatments. The preliminary results reported in the present study suggest that the analysis of walking test including $360^{\circ}$ turn using a single inertial sensor is a promising solution for analyzing FoG mechanism in PD population.

\section{ACKNOWLEDGMENT}

We thank Graham Harker and Georgeann Booth for assistance with data collection.

\section{REFERENCES}

[1] O. Moore, C. Peretz, and N. Giladi, "Freezing of gait affects quality of life of people with Parkinson's disease beyond its relationships with mobility and gait," Mov. Disord., vol. 22, no. 15, pp. 2192-2195, 2007.

[2] J. G. Nutt, B. R. Bloem, N. Giladi, M. Hallett, F. B. Horak, and A. Nieuwboer, "Freezing of gait: moving forward on a mysterious clinical phenomenon," Lancet Neurol., vol. 10, no. 8, pp. 734-744, 2011.

[3] A. H. Snijders, C. A. Haaxma, Y. J. Hagen, M. Munneke, and B. R. Bloem, "Parkinsonism and Related Disorders Freezer or non-freezer: Clinical assessment of freezing of gait," Park. Relat. Disord., vol. 18, no. 2, pp. 149-154, 2012.

[4] N. Giladi and A. Nieuwboer, "Understanding and treating freezing of gait in parkinsonism, proposed working definition, and setting the stage," Mov. Disord., vol. 23, no. S2, pp. S423-S425, Jul. 2008.

[5] A. H. Snijders, M. J. Nijkrake, M. Bakker, M. Munneke, C. Wind, and B. R. Bloem, "Clinimetrics of freezing of gait," Mov. Disord., vol. 23, no. SUPPL. 2, pp. 468-474, 2008.

[6] A. Bengevoord, et al., "Center of mass trajectories during turning in patients with Parkinson' s disease with and without freezing of gait," Gait \& Posture, vol. 43, pp. 54-59, 2016.

[7] H. Bhatt, F. Pieruccini-faria, and Q. J. Almeida, "Parkinsonism and Related Disorders Dynamics of turning sharpness in fl uences freezing of gait in Parkinson 's disease," Park. Relat. Disord., vol. 19, no. 2, pp. 181-185, 2013.

[8] J. Spildooren, S. Vercruysse, K. Desloovere, W. Vandenberghe, E. Kerckhofs, and A. Nieuwboer, "Freezing of Gait in Parkinson', s Disease : The Impact of Dual-Tasking and Turning," Mov. Disord., vol. 25, no. 15, pp. 2563-2570, 2010.

[9] M. Mancini, K. Smulders, R. G. Cohen, F. B. Horak, N. Giladi, and J. G. Nutt, "The clinical significance of freezing while turning in Parkinson's disease," Neuroscience, vol. 343, pp. 222-228, 2017.

[10] A. Burleigh-jacobs, F. B. Horak, J. G. Nutt, and A. O. Jose, "Step Initiation in Parkinson's Disease : Influence of Levodopa and External Sensory Triggers," Mov. Disord., vol. 12, no. 2, pp. 206-215, 1997.

[11] J. Nantel, C. de Solages, and H. Bronte-Stewart, "Repetitive stepping in place identifies and measures freezing episodes in subjects with Parkinson's disease," Gait \& Posture, vol. 34, no. 3, pp. 329-333, 2011.

[12] F. Horak, L. King, and M. Mancini, "Role of Body-Worn Movement Monitor Technology for Balance and Gait Rehabilitation," Phys. Ther., vol. 95, no. 3, pp. 461-470, Mar. 2015.

[13] U. Della Croce, A. Cereatti, and M. Mancini, "Gait Parameters Estimated Using Inertial Measurement Units," in Handbook of Human Motion, B. Müller, et al., Eds. Cham: Springer International Publishing, 2017, pp. 1-21

[14] J. E. Visser, et al., "Quantification of trunk rotations during turning and walking in Parkinson 's disease," Clin. Neurophysiol., vol. 118, pp. 1602-1606, 2007.

[15] C. Zampieri, A. Salarian, P. Carlson-Kuhta, K. Aminian, J. G. Nutt, and F. B. Horak, "The instrumented timed up and go test: potential outcome measure for disease modifying therapies in Parkinson's disease.," $J$. Neurol. Neurosurg. Psychiatry, vol. 81, no. 2, pp. 171-6, 2010.

[16] M. El-Gohary, S. Pearson, J. McNames, M. Mancini, F. Horak, S. Mellone, and L. Chiari, "Continuous Monitoring of Turning in Patients with Movement Disability," Sensors, vol. 14, no. 1, pp. 356-369, 2013.

[17] M. Bertoli, A. Cereatti, D. Trojaniello, A. Ravaschio, and U. Della Croce, "The identification of multiple U-turns in gait: comparison of four trunk IMU-based methods," in Proceedings of the 11th EAI International Conference on Body Area Networks, 2016, pp. 45-48. 